\title{
Numerical Analysis of the Effect of Scanning Speed on the Temperature Field Distribution for Laser Heat Treatment Applications
}

\author{
Furat I. Hussein Al-Najjar \\ Mechatronics Engineering Department \\ Al-Khwarizmi College of Engineering \\ University of Baghdad \\ Baghdad 10071, Iraq. \\ furatnejjar@uobaghdad.edu
}

Received: 26-Nov.-2017 Revised: 25-Dec.-2017 Accepted: 04-Jan.-2018

http://doi.org/10.29194/NJES21020213

\begin{abstract}
One of the unique properties of laser heating applications is its powerful ability for precise pouring of energy on the needed regions in heat treatment applications. The rapid rise in temperature at the irradiated region produces a high temperature gradient, which contributes in phase metallurgical changes, inside the volume of the irradiated material. This article presents a comprehensive numerical work for a model based on experimentally laser heated AISI 1110 steel samples. The numerical investigation is based on the finite element method (FEM) taking in consideration the temperature dependent material properties to predict the temperature distribution within the irradiated material volume. The finite element analysis (FEA) was carried out using the APDL scripting language (ANSYS Parametric Design Language) that is provided by the commercial code ANSYS. Infrared (IR) thermography technique was used to explore the workpiece surface and to validate the obtained results. The work takes into account the effect of different speeds of the laser beam and pulses overlap on the temperature pattern of the material surface and depth.
\end{abstract}

Keywords: Laser heating, Pulsed laser, FEA, Infrared thermography.

\section{Introduction}

Surface hardening, annealing, bending and forming are the most important applications of laser heating in laser materials processing. When a laser beam strikes a metal surface, light absorption occurs within its first few atomic layers and the energy is divided among the reflected, refracted and absorbed parts [1]. The absorbed part of the laser energy is transformed into thermal energy during an interaction time on a time scale less than the pulse duration [2]. For millisecond long pulses, if the intensity of the incident beam is less than $10^{5}$
$\mathrm{W} / \mathrm{cm}^{2}$, the absorbed energy will lead to heating up the solid below its melting temperature. The absorbed energy is lost by conduction, convection, and radiation. This results in a temperature gradient inside the bulk of irradiated material depending on the material thermo-physical properties as well as the laser beam working parameters [3].

Pulsed laser beams in specific are characterized by its temporal and spatial control facility [4]. In laser material processing applications, the possibility of temperature distribution control can be attained through manipulating the temporal and spatial parameters of a laser such as peak power, pulse duration, pulse repetition rate, scanning speed, pulse shape and spot size [5, 6]. In the recent years, much research on laser heating modeling were carried out. Z. Shen et al. [7] applied numerical and analytical models for a heat transfer process due to irradiating a substrate by a moving laser beam. The numerical simulation reveals good results in terms of temperature profiles and time history at the special points. A. Kaplan et al. [8] developed a mathematical model for calculating the temperature dependent absorptivity during pulsed laser heating and melting for a copper substrate coated and galvanized with $\mathrm{Ni}$ and $\mathrm{Au}$. R. Gospavic [9] presented an analytical study for analyzing the temporal and spatial temperature distribution inside a cylindrical geometry of a material due to laser irradiation its upper surface. D. Sowdari et al. [10] developed a numerical model for analyzing the heating rate and temperature distribution in metals during laser heating and melting processes. A limited case analytical model was utilized for validating the computational results. B.S. Yilbas [11] introduced a numerical model for predicting the temperature field and phase change in a cemented car-bide tool during laser heating. The experimental part showed a high temperature gradient along the laser beam axis. Qing Peng [12] used the integral transform method to obtain a general solution for the transient temperature field in a finite slap heated by a moving heat source. An experimental 
part and a FEA were applied for the analytical solution verifications.

This numerical study was devoted to investigate the thermal field that produced due to a pulsed laser heating process. The objective is to predict the temperature values and thermal distribution for AISI 1110 carbon steel sheets with the aid of ANSYS Multiphysics package release 15.0 as an integral part of the work and validate the output by the IR thermography technique results.

\section{Materials and Setup}

A steel sheet grade AISI 1110 of $20 \times 16 \mathrm{~mm}$ dimension and $1 \mathrm{~mm}$ thickness was used in this investigation. As shown in Figure 1, the specimen is clamped and placed on an insulator sheet to avoid thermal quenching during the heating and cooling cycles.

The metal sheets were irradiated in a single pass with a $1.064 \mu \mathrm{m}$ wavelength pulsed Nd:YAG laser type Han's Laser (China) of the following specifications: $0.1-50 \mathrm{~ms}$ range pulse duration, $200 \mathrm{~Hz}$ maximum pulse repetition rate, $90 \mathrm{~J} / 10 \mathrm{~ms}$ maximum pulse energy and $8 \mathrm{~kW}$ maximum peak power. The laser head is automated with a robotic arm (Motoman, Japan) equipped with a visual camera for track monitoring.

During laser irradiation, the interaction zone was covered with a tent of argon gas to prevent contaminations and to minimize the oxidation effect. The thermal field on the specimen upper surface was monitored with infrared thermography technique. A thermal IR camera type FLIR T-335 was placed over the top surface of the specimen to observe the interaction zone during the process.

\section{Mathematical Formulation 3.1 Laser Heat Source}

In laser heating of solids, the laser beam is assumed as a Gaussian distribution heat source whose intensity and shape vary with the penetration depth and beam radius:

$q(r)=\frac{f(1-R) P}{\pi r_{o}^{2}} \exp \left(-f\left(\frac{x}{r_{o}}\right)^{n 1}-f\left(\frac{y}{r_{o}}\right)^{n 2}\right)$

where $x$ and $y$ are the spatial coordinates on the surface $(\mu \mathrm{m}), R$ is the surface reflectivity, $P$ is the laser beam power $(\mathrm{W}), r_{o}$ is the initial radius $(\mathrm{m}), f$ is the coefficient that characterizes the beam distribution (usually equal to 3 ) and $n_{1}=n_{2}=2$ for Gaussian distribution.

In modeling the laser beam for heating applications, it is difficult to predict with high accuracy the size and shape of the heat source along the depth of a solid. It can be assumed as a Gaussian volumetric source of given radius and height with homogenous power distribution inside:

$q_{v}(r . z)=\frac{f(1-R) P}{\pi r_{o}^{2} d} \exp \left(-\frac{r^{2}}{r_{o}^{2}}\right) u(z) \quad \cdots \ldots(2)$

where $d$ is the material thickness $(\mathrm{mm}), z$ is the current depth along $d, r$ is the radius for the current depth $=\sqrt{x^{2}+y^{2}}(\mathrm{~m})$ and $u(z)=1$ for $0<z<d$ and $u(z)=0$ for other positions.

In fact, the beam power and energy decreases along the penetration depth in a linear manner. Often, it is assumed a linear decrease of energy may take an overturned cone shape inside the solid bulk $[13,14]$ :
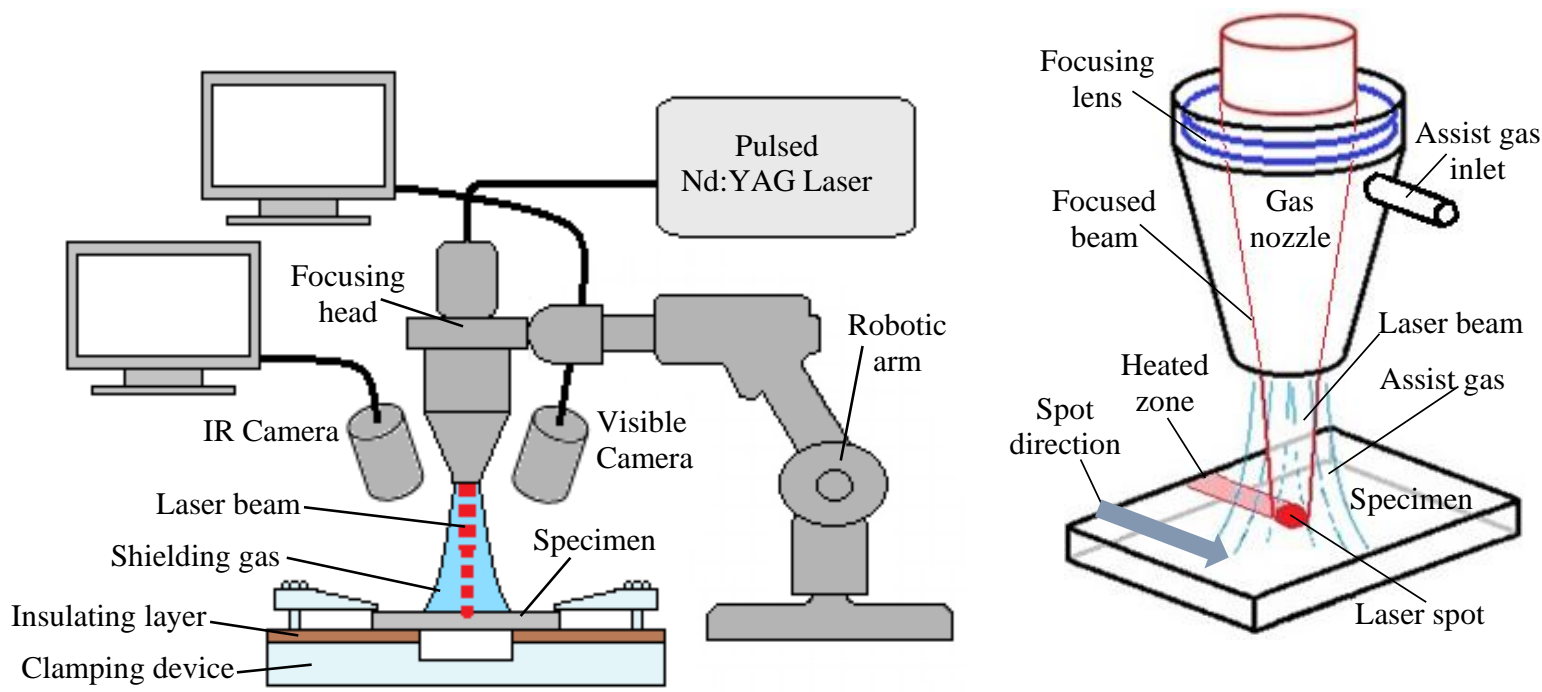

Figure 1:

Experimental setup schematic diagram (not in scale). 
$q_{v}(r . z)=\frac{f P}{\pi r_{o}^{2} d} \exp \left(\left(1-\frac{r^{2}}{r_{o}^{2}}\right)\left(1-\frac{z}{d}\right)\right) \ldots \ldots$ (3)

Some losses occur to the incident laser beam power due to the surface reflectivity, therefore the laser beam intensity can be expressed as [15]:

$I=\frac{4(1-R) P}{\pi r^{2}}$

where $I$ is the laser beam intensity on the material surface.

\subsection{Temperature Field}

Applying a moving heat source in a certain path on a solid body would yield thermal contours propagates in changeable profiles with time through the material. This can be mathematically modeled with the three-dimensional heat conduction equation [16]:

$$
\begin{gathered}
\rho C\left(\frac{\partial T}{\partial t}-v \frac{\partial T}{\partial x}\right)=\frac{\partial}{\partial x}\left(K \frac{\partial T}{\partial x}\right)+\frac{\partial}{\partial y}\left(K \frac{\partial T}{\partial y}\right)+\frac{\partial}{\partial z}\left(K \frac{\partial T}{\partial z}\right) \\
+Q(\text { x.y.z.t) } \cdots . . .(5)
\end{gathered}
$$

where $T$ is the absolute temperature, $t$ is the time, $x, y$ and $z$ are the Cartesian coordinates, $K$ is the thermal conductivity, $\rho$ is the density, $C$ is the specific heat, $v$ is the scanning speed and $Q$ is the rate at which heat is supplied to the solid per unit time and volume.

The left term represents the quantity of deposited energy within the material, which represents the change in enthalpy. In laser heating applications, for heat treatment applications as an example, it is assumed a point heat source moves on a thick plate and involves three-dimensional heat flow. The heat source $Q$ as a function of $z$ can be expressed by [17]:

$Q=-\frac{I(z)}{\partial z}$

where $I(z)$ is the intensity of the incident laser beam as a function of depth $z$.

For Gaussian distribution of the optical energy across within the incident beam on a metal surface, the intensity and energy distribution vary along the radius and depth:

$Q(x, y, z)=I_{o} \alpha e^{\left(-\frac{r^{2}}{r_{o}^{2}}\right)}$

where $I_{o}$ is the incident intensity at the surface, $\alpha$ is the absorption coefficient, $r_{o}$ is the spot radius and $r$ is a radial position inside the spot which can be determined by from $r=\sqrt{x^{2}+y^{2}}$.
The incident optical energy penetrates to a depth equal to reciprocal of the absorption coefficient:

$$
l_{o p}=\frac{1}{\alpha}=\frac{\lambda}{4 \pi k}
$$

where $l_{\mathrm{op}}$ is the optical penetration depth, $\lambda$ is the laser wavelength and $k$ is the extinction coefficient.

To achieve clean scanning of the laser spot over the surface by blowing the produced contamination and prevent surface oxidation, the laser heating process may involves applying assisting gas. In addition to the convection mechanism, to achieve accurate analysis the radiation mechanism was involved too [18]:

$-K \frac{d T}{d n}=\sigma \epsilon\left(T_{s}^{4}-T_{a}^{4}\right)+h c\left(T_{s}-T_{a}\right) \quad \ldots \ldots(9)$

where $\mathrm{n}$ is the normal outward vector to the surface of specimen, $\sigma$ is the Stefan Boltzmann constant, $\varepsilon$ is the emissivity, $T s$ is the surface temperature, $T_{a}$ is the ambient temperature and $h c$ is surface heat transfer coefficient due to the forced convection of cooling gas.

The initial conditions for transient analysis is the temperature $(T)$ of the body is equal to ambient temperature:

$T(x . y . z .0)=T_{o}(x . y \cdot z) \quad \ldots \ldots(10)$

where $T_{o}$ is the ambient temperature $(\mathrm{K})$.

\section{Temperature Field}

The finite element ANSYS package Release 15.0 along with many subroutines are used to simulate the FEM thermal process of laser heating. The main steps and important procedures of the simulation process are presented in a flowchart shown in Figure 2.

\subsection{Creating the Model and Mesh Generation}

A flat plate was created through top down mode geometry codes with model dimensions to simulate the workpiece in real sizes. FEA is highly related with the mesh density, where coarse elements affect the simulation in term of accuracy, on contrary extreme dens mesh increases the processing time with no noticeable accuracy enhancement. To find the optimum mesh that gives a trusted solution, many trials were carried out by irradiating a sample and calculate the maximum temperature for a specified node at different mesh densities. The optimum density is selected when temperature starts to settle down at a constant value as illustrated in Figure 3. The built models are 
meshed using SOLID 70 elements type which have eight nodes and single degree of freedom.

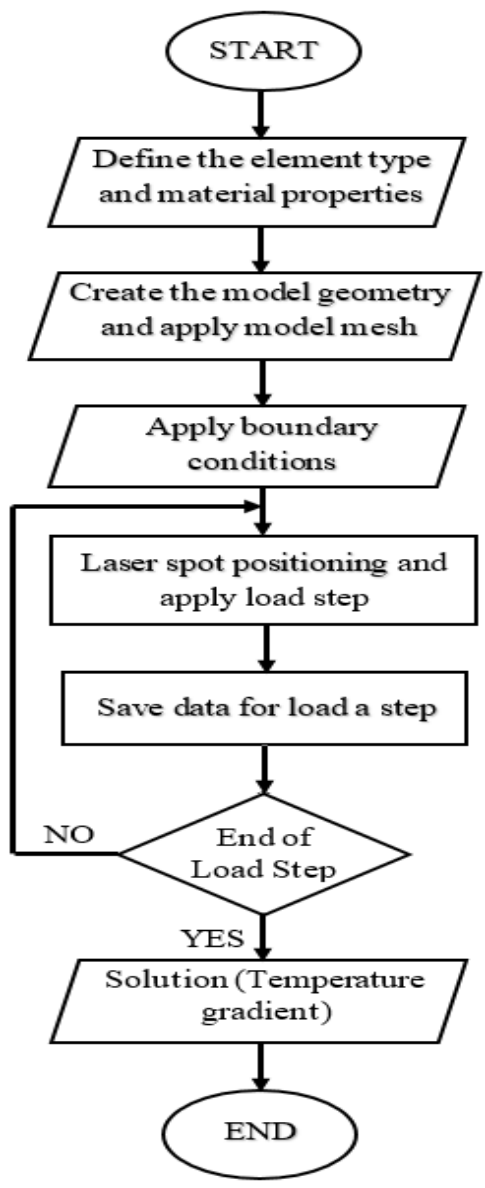

Figure 2:

Laser heating simulation flowchart.

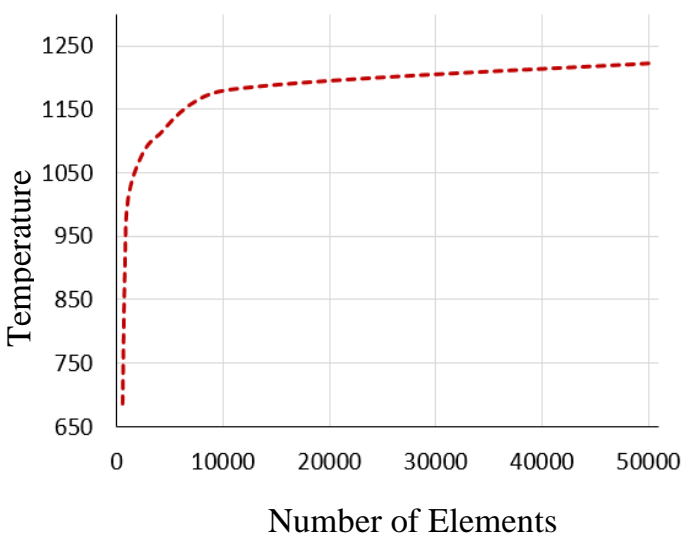

Figure 3:

Obtaining the optimum mesh is crucial in FEA simulation accuracy.

\subsection{Material Properties}

The simulation was fed with the necessary information of the thermo-physical material properties that define the processed metal. Figure
4 illustrates the thermal conductivity, specific heat, thermal diffusivity and mass density temperature dependent properties that used as inputs for accurate thermal modeling investigation [19-21]. The carbon steel shows a good reflectivity of about 0.6 to the Nd:YAG $1064 \mathrm{~nm}$ wavelength [22]. This value dose not suffer a noticeable change when the temperature varies within the range between $500 \mathrm{~K}$ to $1500 \mathrm{~K}$ [1].
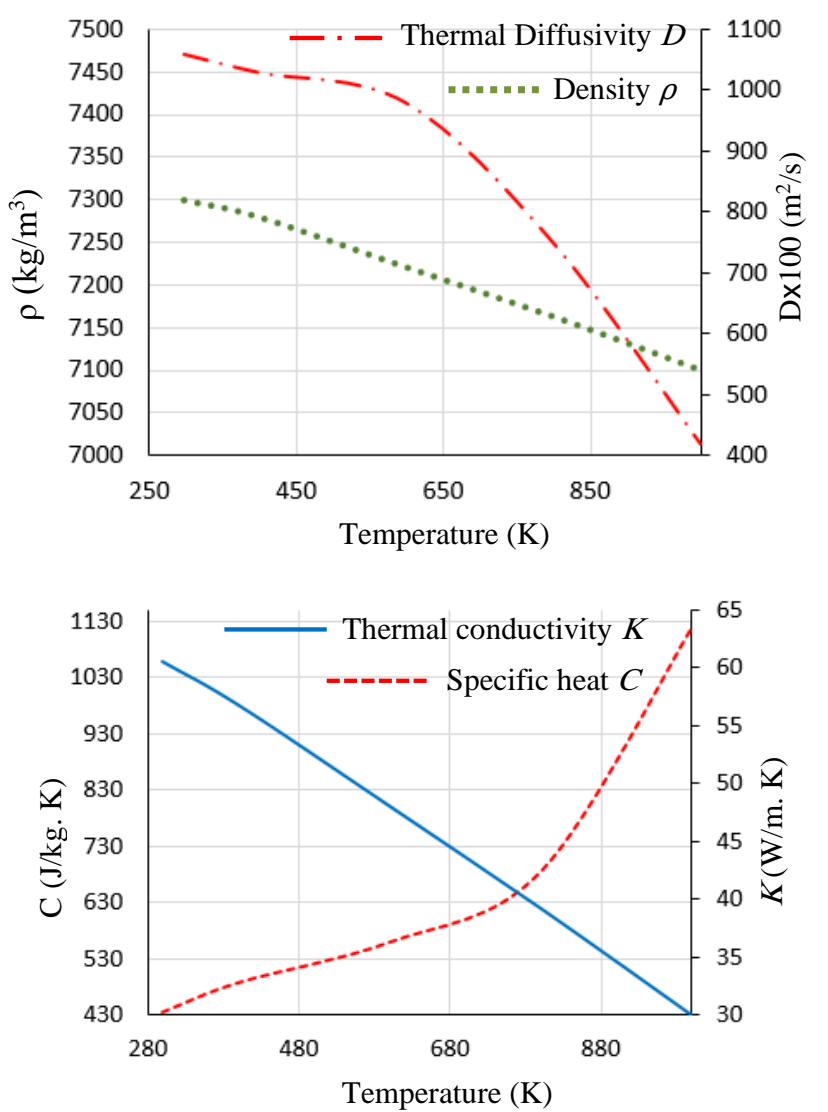

Figure 4:

Thermo-physical properties of carbon steel AISI 1110 [19-21]

\subsection{Simulation Assumption}

When the simulation is developed, the following assumptions were taken into account:

- The laser beam has a Gaussian distribution TEM $_{00}$ mode (Figure 5).

- The process includes heating without phase physical change (but metallurgical change is possible).

- The irradiated material is homogenous and isotropic.

- There is no heat transfer from the workpiece to the clamps and base.

- All the heating, cooling and boundary conditions are symmetrical across the laser spot propagation line on the workpiece. 
- The thermal properties are temperature dependent.

- The following information are assumed in simulation: the workpiece initial temperature is 30 ${ }^{\circ} \mathrm{C}$, the convection coefficient is $25 \mathrm{~W} / \mathrm{m}^{2}{ }^{\circ} \mathrm{C}$ and steel emissivity value for is 0.25 .

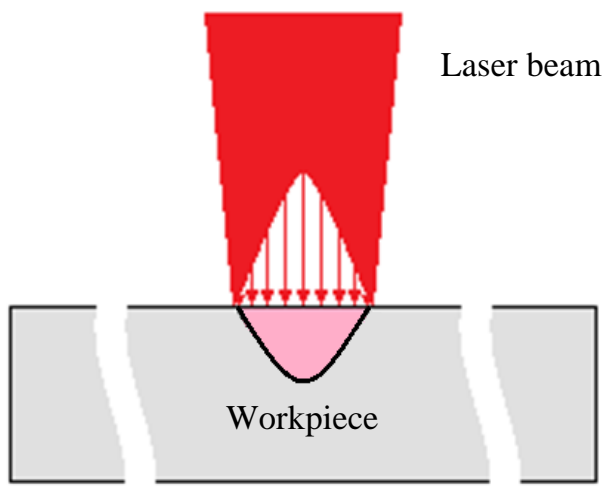

Figure 5:

Gaussian distribution laser beam

of $\mathrm{TEM}_{00}$ mode.

\subsection{Working Parameters}

Laser heating is a multi-input/output process, where the input parameters play an important role in plotting the temperature field profile. Many APDL subroutines are used to study the effect of varying the scanning speed parameter on the heating process. As mentioned earlier, the objective of the current work is to explore the thermal field on the surface and predict it within bulk of the workpiece. Accordingly, four sets of different scanning speeds were considered as presented in Table 1.

Table 2:

Experiment variables and constant parameters.

\begin{tabular}{|c|c|}
\hline Pulse duration $(\mathrm{ms})$ & 3 \\
\hline Peak power $(\mathrm{kW})$ & 2 \\
\hline Scanning speed $(\mathrm{m} / \mathrm{s})$ & 1 to 4 \\
\hline Pulse repetition rate $(\mathrm{Hz})$ & 15 \\
\hline Spot size $(\mu \mathrm{m})$ & 500 \\
\hline gas pressure $(\mathrm{bar})$ & 1 \\
\hline
\end{tabular}

\section{Results and discussion}

Laser heating via a moving laser beam on the steel surface is considered and the gradual change in temperature without physical phase change is modeled. Here, the influence of changing the scanning speed on the temperature field was examined.

It is difficult to employ a transducer to measure the temperature inside the bulk of the metal, on the other hand the IR thermal measurement can cover the outer metal surfaces. Therefore, the IR thermal images may be useful to grant validation and authenticity for the obtained simulation results inside the material bulk as well as the surface. Figure 6 shows how does it done, a comparison between the experimental images that obtained by the IR thermal camera and that obtained from the simulation work. The comparison was done at the upper surface, $x-y$ at $z=0$ plane along the linear heating path when the laser spot at the middle position $(x=10 \mathrm{~mm})$ from the start point. The used pulsed Nd:YAG laser (1064 nm wavelength) working parameters are $2 \mathrm{~ms}$ pulse duration, $15 \mathrm{~Hz}$ pulse repetition rate, $2 \mathrm{~kW}$ peak power, $60 \mathrm{~W}$ average power and $4 \mathrm{~m} / \mathrm{s}$ scanning speed. The result shows an acceptable agreement of temperature values and profiles for both readings. The calculated error between the actual values of experimental part and the estimated simulation results is about $5.5 \%$ which allows for considering the obtained results from the FEM reliable. By examining the IR image in the figure, it can be seen that the maximum intensity is in the center of the spot of irradiated laser and reduced radially out of spot and this reflects the Gaussian distribution of the laser power.

Under the same working parameters, Figure 7 shows a comparison between the experimental and estimated values along the heating path $x$-axis at the middle of the metal sheet.

Figure 8 shows a three-dimensional temperature distribution in the cross section of the metal sheet, through $x$-axis, at the middle path under the same working parameters of Figure 5. As seen the laser spot is focused at the upper surface $(z=0)$ and propagates along the heating path $(x-$ axis) to plot the temperature contours. The red zone reveals the highest temperature region where the laser and the metal interact, later the heat diffuses out in the direction of the temperature gradient. The interaction zone takes an elliptical shape and can be represented as the volumetric Gaussian heat source for the metal bulk. Due to laser spot moving, the interaction zone moves and the temperature gradient translates, as a result another elliptical contour extend due to the motion of the heat source, the new heated regions in the previous load steps and the cooling stroke.

For laser heat treatment applications, the working parameters should be set to avoid metal melting, evaporation, or may be increase the surface roughness. One of most effective and controllable parameters is the scanning speed [23]:

$E_{a}=\frac{I \cdot \tau \cdot P R R}{v \cdot r} \quad \ldots \ldots(11)$

where, $E_{a}$ is the average supplied energy per load step $\left(\mathrm{J} / \mathrm{m}^{2}\right), \tau$ is the pulse duration $(\mathrm{ms}), P R R$ is the pulse repetition rate, $v$ is the scanning speed $(\mathrm{m} / \mathrm{s})$ and $r$ is the spot size $(\mu \mathrm{m})$. 


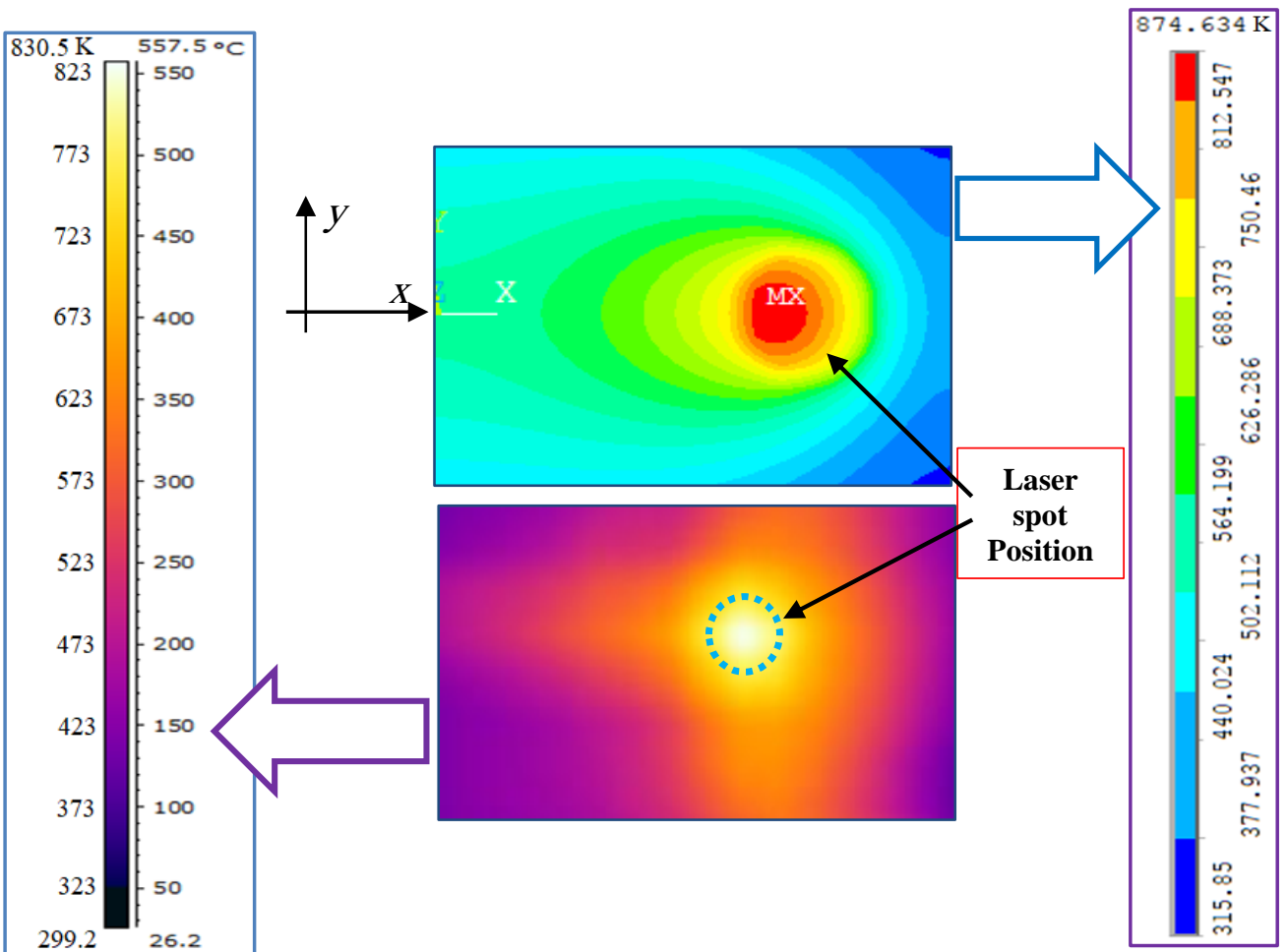

Figure 6: FEA simulation validation based on comparison with the thermal camera results at the upper surface $x-y$ plane when the spot at the middle of the heating path $(x=10)$.

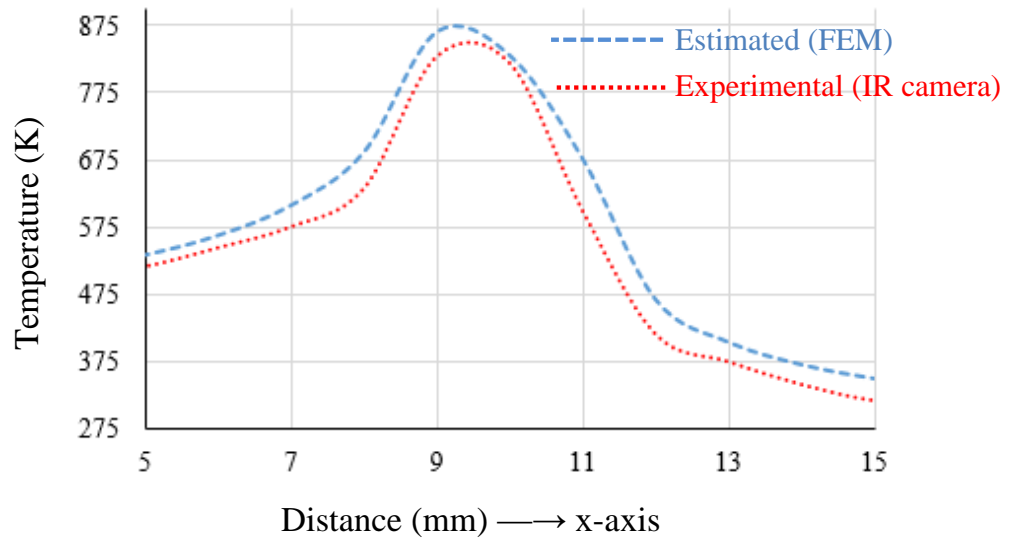

Figure 7:

A comparison between the actual and estimated values along the heating path $\mathrm{x}$-axis at the middle of the metal sheet.

According to Equation 11, the rate of heat transfer between the laser and the metal at the interaction zone will be high for low speeds and vice versa. Furthermore, the size of interaction zone as a volumetric heat source for the bulk will be less. Actually, the above factor is highly correlated with repetitive pulse overlap percentage $(P O)$ which defines the number of laser shots per one load cycle $(N)$ [23]:

$$
N=1-\frac{1}{(1-P O)}
$$

where,

$$
P O=1-\frac{v}{P R R(r+v \cdot \tau)}
$$




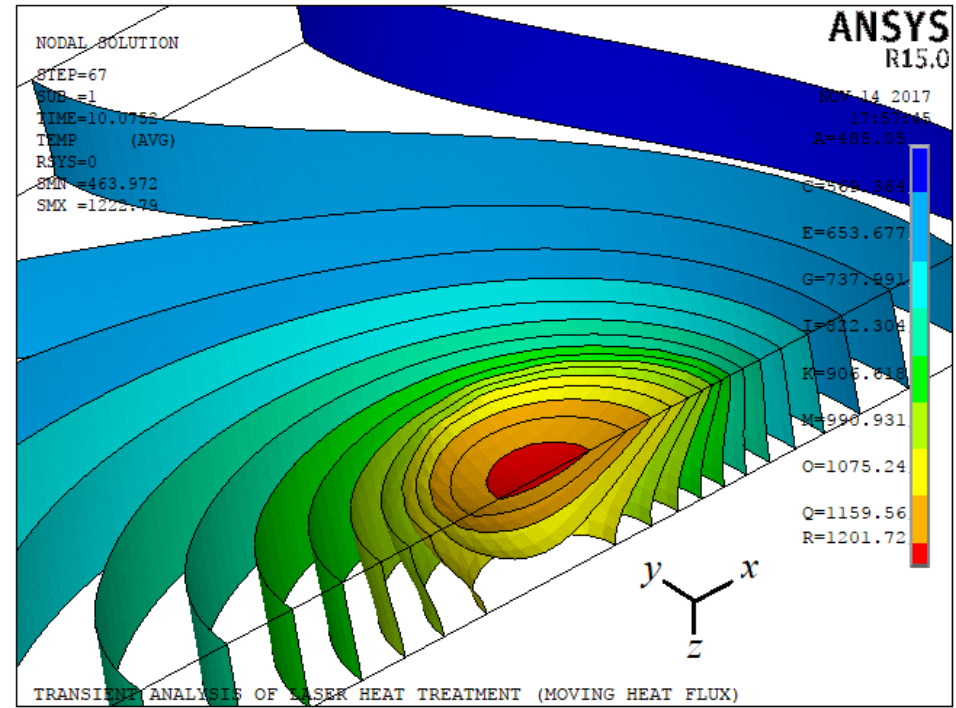

Figure 8:

The temperature distribution within the metal sheet bulk.

Figure 9 shows the temperature profile along $x$ axis for the four used speeds values. It is obvious at lower speeds the heating effect is higher and this is true for the other axes. That means that the interaction zone volume is related with the scanning speed which controls the heating process, as well as other working parameters. The workpiece length starts at the beginning of $x=0 \mathrm{~mm}$ and ends when $x=20 \mathrm{~mm}$.
It can be seen that the maximum temperature value is at a position slightly behind the laser spot. In addition, the temperature drops more sharply in the front of the laser spot zone than the behind laser spot zone where the decay occurs gradually. This is related to the quenching effect of cold part of the unprocessed region in front of the spot. It is obvious from the graph the processed region behind the spot is approximately at constant temperature for a period.

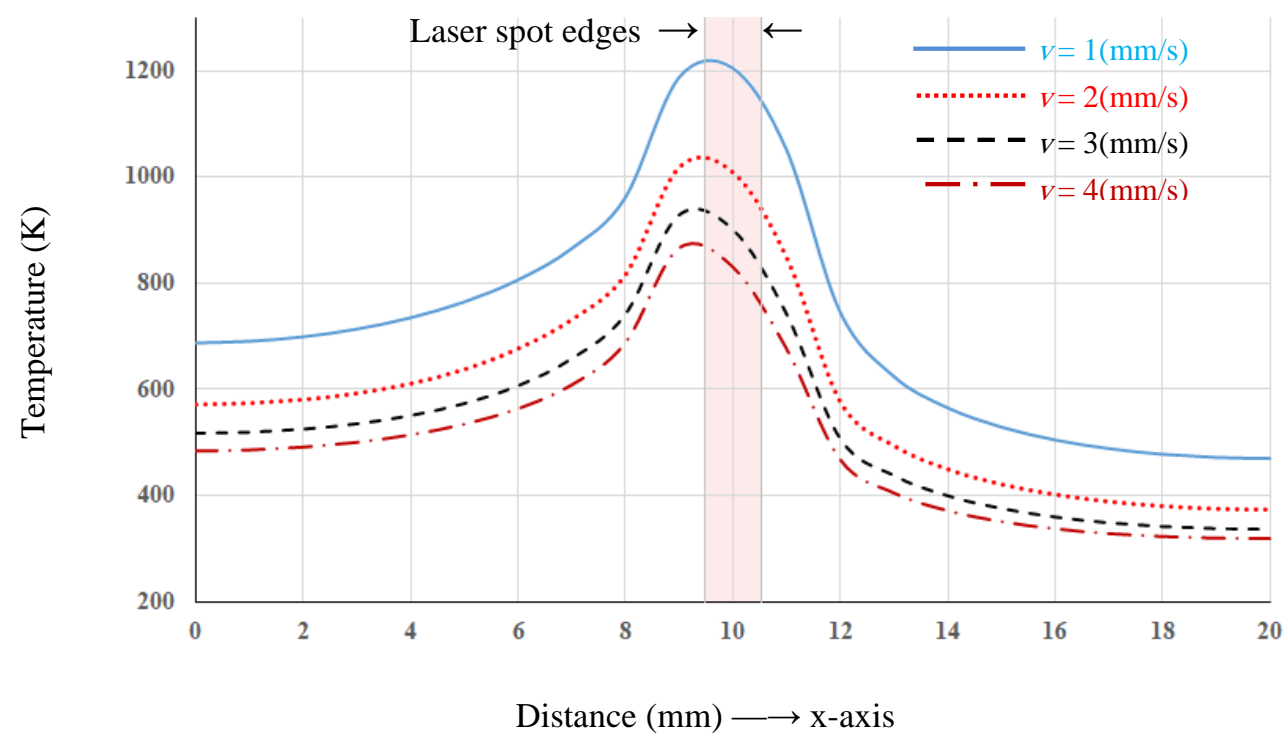

Figure 9:

Temperature profiles along the heating path $\mathrm{x}$-axis for four speeds at working parameters of $2 \mathrm{~kW}$ peak power, $3 \mathrm{~ms}$ pulse duration, 15 pulse repetition rate. 
Figure 10 shows the temperature distribution along the $y$-axis at the same position of the laser spot $(x=10)$ and the same working parameters (as in Figure 8) for the used four speeds. The temperature decays gradually out of laser spot center $(y=0)$ to a region adjacent to the laser spot edge $(y=1 \mathrm{~mm})$. After this position the temperature decays in a sharp manner for a wider temperature gradient. It can be noticed that the temperature profiles for all speeds share the same pattern although the temperature values are significantly different and this is true for other axes.
Figure 11 shows temperature distribution along the $z$-axis at the same position of the laser spot $(x=10)$ and the same working parameters (as in Figures 8 and 9) for the four speeds. Here, the pulse overlap $P O$ plays a major in heating the metal bulk in depth. It is evident that the temperature contours extend into the metal depth with similar profiles when the scanning speed decreases. The Gaussian distribution of the laser spot at the surface $(z=0)$ build a temperature gradient along the $z$-axis.

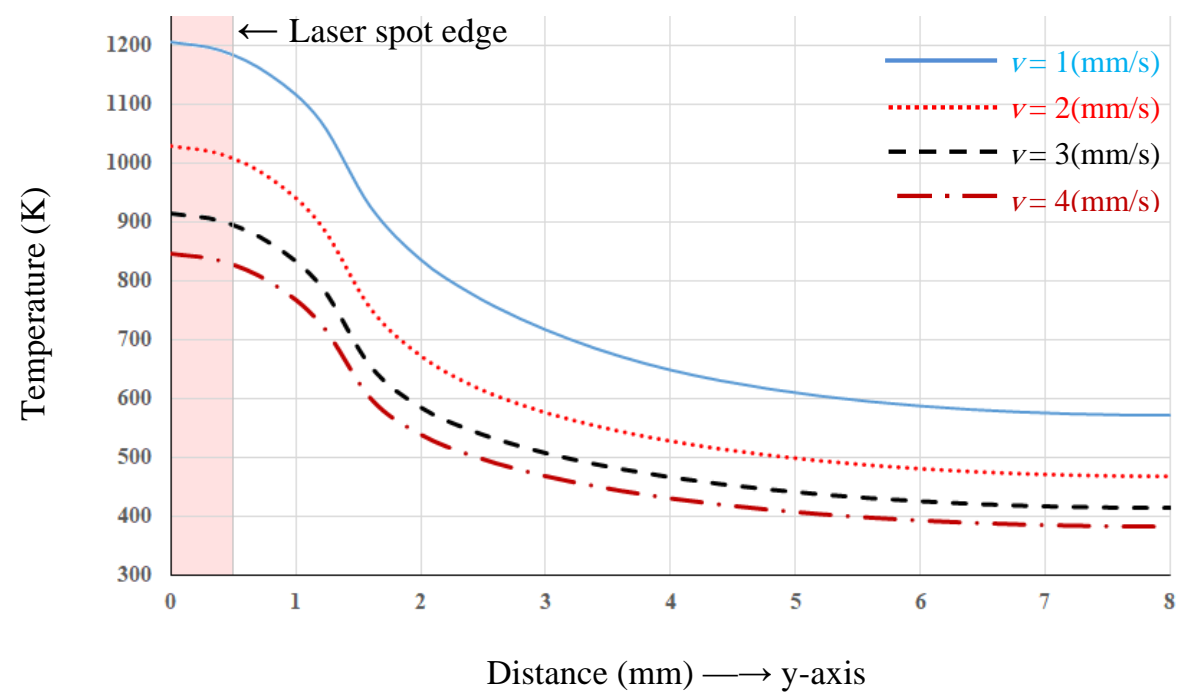

Figure 10: Temperature profiles for the half-space sheet along the $y$-axis for four speeds at working parameters of $2 \mathrm{~kW}$ peak power, $3 \mathrm{~ms}$ pulse duration, 15 pulse repetition rate.

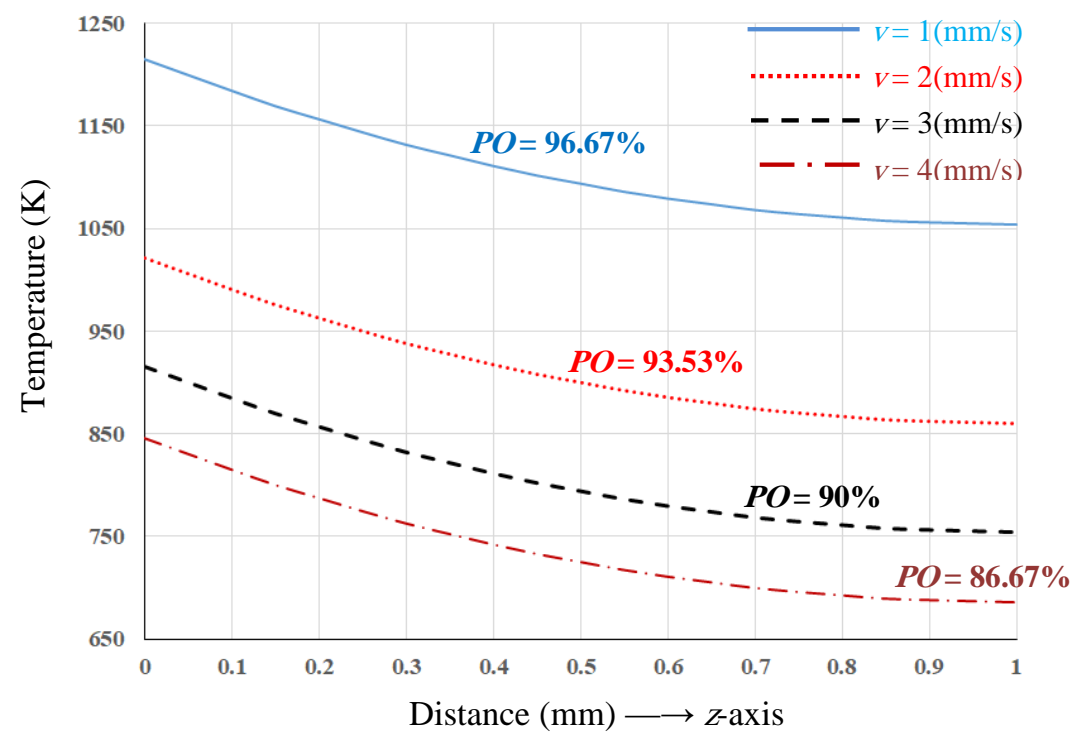

Figure 11: Temperature profiles along the z-axis for four speeds at working parameters of $2 \mathrm{~kW}$ peak power, $3 \mathrm{~ms}$ pulse duration, 15 pulse repetition rate. 


\section{Conclusions}

A numerical model based on finite element method is developed to analyze the temperature distributions within a steel sheet irradiated by a moving laser beam. The study shows the significance of the scanning speed on the heating effect, which may be of value for heat treatment applications. The experimental part represented by IR thermal imaging results donates the simulation results validation in an accuracy of $5.5 \%$. This paper has clearly showed the following remarks:

- Modeling laser material processing application can be utilized for predicting the temperature field especially for difficult detectable issues.

- Study the effect of one of the working parameters (here the scanning speed) is essential laser heat treatment applications optimization and metallurgical phase changing understanding.

- In laser heat treatment the interaction zone between laser and the metal represents optical/thermal exchange region and the volumetric heat source for the processed metal. The temperature and shape of this zone outline the temperature values and distribution manner inside the bulk.

- The surface temperature and thermal penetration can be altered by adjusting the proper scanning speed.

- In lateral heating along the (y-axis) the temperature drops in a sharp manner. Therefore, for wide surface treatment multi-passes is required to scan the whole surface.

- Control the heating depth is correlated with the pulse overlap percentage which is related to the scanning speed.

\section{Acknowledgments}

This work was supported by the Mechatronics Engineering Department, Al-Khwarizmi College of Engineering, University of Baghdad and Institute of Laser for Postgraduate Studies, University of Baghdad.

\section{References}

[1] W. M. Steen. "Laser Material Processing" $4^{\text {th }}$ edition. Springer London Dordrecht Heidelberg New York, 2010.

[2] M. Allmen, A. Blatter. "Laser-Beam Interactions with Materials: Physical Principles and Applications" $2^{\text {nd }}$ edition. Springer-Verlag Berlin Heidelberg GmbH, 1998.

[3] J. Dutta Majumdar and I. Manna. "Laser Processing of Materials". Sadhana, 2003; 28, Parts $3 \& 4: 95-562$.
[4] Yih-Fong Tzeng. "Effects of operating parameters on surface quality for the pulsed laser welding of zinc-coated steel". Journal of Materials Processing Technology, Elsevier. 100 (2000) 163170.

[5] F. Hussein, E. Akman , B. Oztoprak, M. Gunes, O. Gundogdu, E. Kacar, K. Hajim and A. Demir. "Evaluation of PMMA joining to stainless steel 304 using pulsed Nd:YAG laser", Elsevier. Optics \& Laser Technology 49(2013)143-152.

[6] K.A. Mumtaz and N. Hopkinson. "Selective Laser Melting of thin wall parts using pulse shaping". Journal of Materials Processing Technology, Elsevier. 210 (2010) 279-287.

[7] S. Shuja, B. Yilbas and O. Momin." Laser heating of a moving slab: Influence of laser intensity parameter and scanning speed on temperature field and melt size". Optics and Lasers in Engineering, Elsevier. 49(2011)265-272.

[8] A. Kaplan. "Model of the absorption variation during pulsed laser heating applied to welding of electronic Au/Ni-coated Cu-lead frames". Applied Surface Science 241 (2005) 362-370.

[9] R. Gospavic, M. Sreckovic, V. Popov and Goran Todorovic. "3D modeling of material heating with the laser beam for cylindrical geometry". Mathematical and Computer Modelling 43 (2006) 620-631.

[10] D. Sowdari and P. Majumdar. "Finite element analysis of laser irradiated metal heating and melting processes". Optics \& Laser Technology 42 (2010) 855-865.

[11] B. Yilbas, S. Shuja and S. Khan. "Laser repetitive pulse heating of tool surface" Optics \& Laser Technology 43 (2011) 754-761.

[12] Q. Peng. "An analytical solution for a transient temperature field during laser heating a finite slab" Applied Mathematical Modelling 000 (2015) 1-7.

[13] W. Piekarska, M. Kubiak and Z. Saternus. "Application of Abaqus to analysis of the temperature field in elements heated by moving heat sources". Archives of foundry engineering volume 10, issue 4/ 2010, 177 - 182.

[14] S. Paul, R. Singh and W. Yan. "Finite Element Simulation of Laser Cladding for Tool Steel Repair". Lasers Based Manufacturing, the $5^{\text {th }}$ International and $26^{\text {th }}$ All India Manufacturing Technology, Design and Research Conference, AIMTDR 2014. 
[15] I. Ivanovi, A. Sedmak, M. Miloš, A. Živkovi and M. Lazi. "Numerical Study of Transient ThreeDimensional Heat Conduction Problem with a Moving Heat Source". Thermal Science, volume 15 , issue $1 / 2011,257-266$.

[16] J. Yanga, S. Suna, M. Brandtb and W. Yanc. "Experimental investigation and 3D finite element prediction of the heat affected zone during laser assisted machining of Ti6Al4V alloy". Journal of Materials Processing Technology, Elsevier. 210 (2010) 2215-2222.

[17] N. Shanmugam, G. Buvanashekaran, and K. Sankaranarayanasamy. "Some Studies on Temperature Distribution Modeling of Laser Butt Welding of AISI 304 Stainless Steel Sheets". International Science Index, Mechanical and Mechatron-ics Engineering Vol:7, No:7, 2013 waset.org/Publication/16444.

[18] Z. Huaa and Xu Jiawena. "Modeling and Experimental Investigation of Laser Drilling with Jet Electrochemical Machining". Chinese Journal of Aeronautics 23(2010) 454-460.

[19] Kenneth C Mills. "Recommended values of thermophysical properties for selected commercial alloys". Woodhead Publishing Limited and ASM International, 2002.

[20] F. P. Incropera and D. P. Dewitt. "Fundamentals of Heat and Mass Transfer", $7^{\text {th }}$ edition. John Wiley \& Sons, Inc.

[21] http://www.matweb.com.

[22] E. Kennedy, G. Byrne and D.N. Collins. "A Review of the Use of High Power Diode Lasers in Surface Hardening". Journal of Materials Processing Technology 155-156 (2004) 18551860.

[23] Y.-F. Tzeng. "Process Characterization of Pulsed Nd:YAG Laser Seam Welding”. Advanced Manufacturing Technology, 2000; 16:10 - 18. 


\title{
التحليل العددي لتأثثير سرعة الليزر على توزيع المجال الحراري

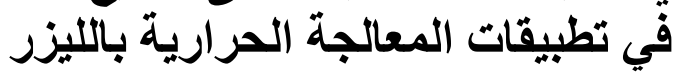

\author{
فرات ابراهيم حسين النجار \\ قسم هندسة الميكاترونكس التئس

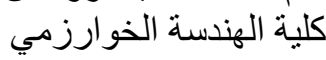 \\ جامعة بغداد
}

furatnejiar@uobaghdad.edu

\section{الخلاصة}

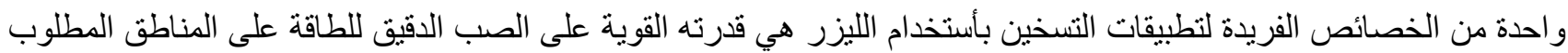

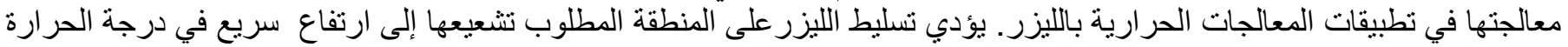

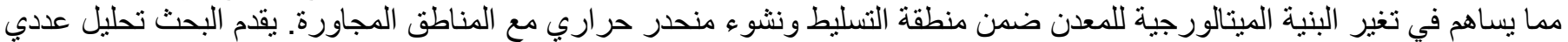

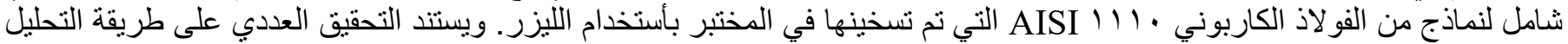

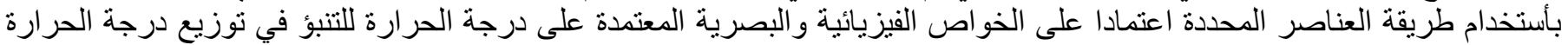

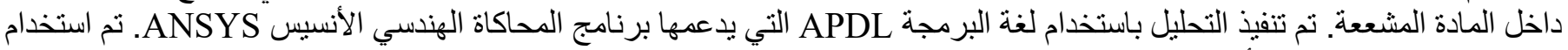

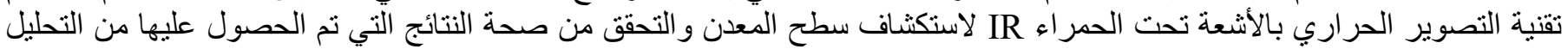

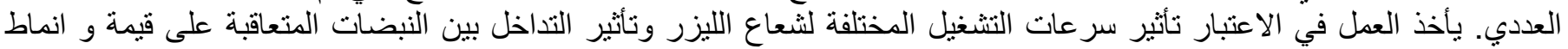
التوزيع الحر اري على سطح المعدن و عمقها. 